\title{
A Novel Method for Rapid Mapping of the Spatial Intensity of Influenza Epidemics
}

\author{
David J. Muscatello ${ }^{1}$, Robert N. Leong ${ }^{1}$, Robin M. Turner ${ }^{2}$, Anthony T. Newall ${ }^{1}$ \\ ${ }^{1}$ School of Public Health and Community Medicine, UNSW Sydney, UNSW Sydney, New South Wales, Australia, ${ }^{2}$ University of Otago, Dunedin, New \\ Zealand
}

\section{Objective}

Using the epidemic of influenza type A in 2016 in Australia, we demonstrated a simple but statistically sound adaptive method of automatically representing the spatial intensity and evolution of an influenza epidemic that could be applied to a laboratory surveillance count data stream that does not have a denominator.

\section{Introduction}

Surveillance of influenza epidemics is a priority for risk assessment and pandemic preparedness. Mapping epidemics can be challenging because influenza infections are incompletely ascertained, ascertainment can vary spatially, and often a denominator is not available. Rapid, more refined geographic or spatial intelligence could facilitate better preparedness and response.

\section{Methods}

Weekly counts of persons with laboratory confirmed influenza type A infections in Australia in 2016 were analysed by 86 substate geographical areas. Weekly standardised epidemic intensity was represented by a z-score calculated using the standard deviation of below-median counts in the previous 52 weeks. A geographic information system was used to present the epidemic progression.

\section{Results}

There were 79,628 notifications of influenza A infections included. Of these, 79,218 (99.5\%) were allocated to a geographical area. The maps indicated areas of elevated epidemic intensity across Australia by week and area, that were consistent with the observed start, peak and decline of the epidemic when compared with weekly counts aggregated at the state and territory level. An example is shown in Figure 1.

\section{Conclusions}

The methods could be automated to rapidly generate spatially varying epidemic intensity maps using a surveillance data stream. This could improve local level epidemic intelligence in a variety of settings and for other diseases. It may also increase our understanding of geographic epidemic dynamics.

\section{Acknowledgement}

We thank the Australian Department of Health for providing the influenza laboratory data used in the study. 


\section{September 2016}
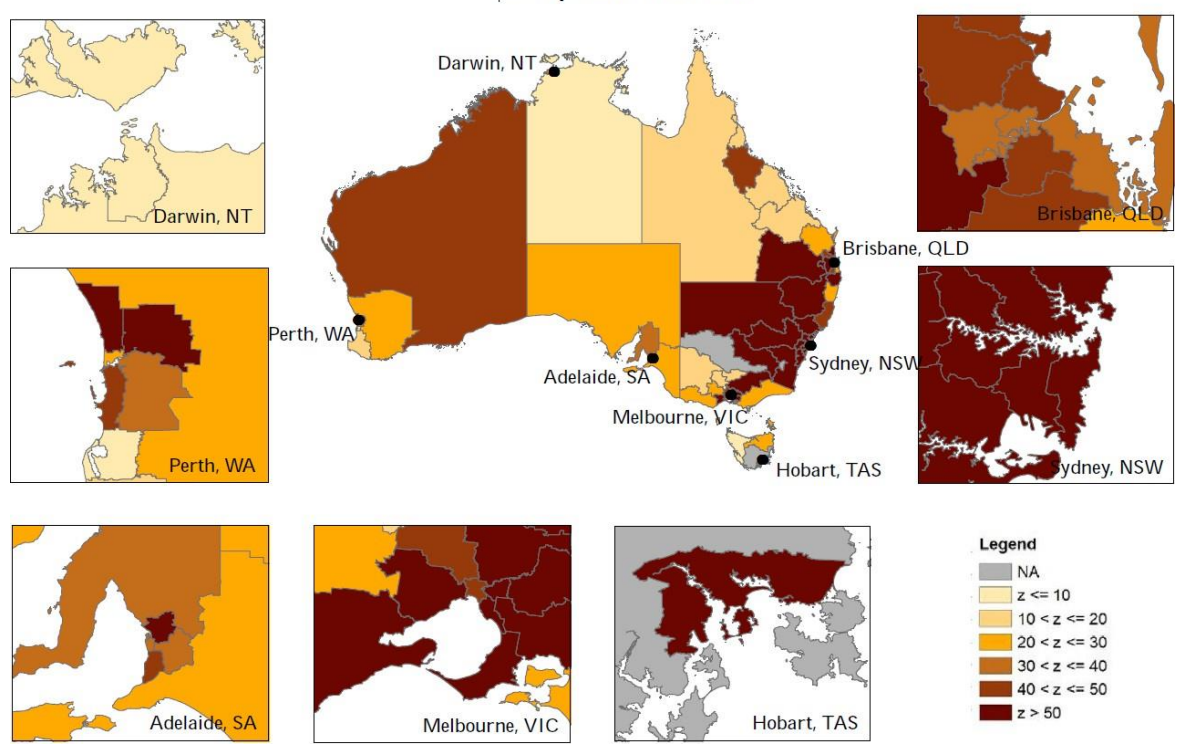

Figure 1. Example map as at 9 September 2016, showing intensity by area during the peak week of the season. Insets show capital cities 